Preprint HU-EP-06/45

\title{
Restoration of Dynamically Broken Chiral and Color Symmetries for an Accelerated Observer
}

\author{
D. Ebert ${ }^{1}$ and V. Ch. Zhukovsky ${ }^{2}$ \\ 1 Institut für Physik, Humboldt-Universität zu Berlin, 12489 Berlin, Germany and \\ 2 Faculty of Physics, Department of Theoretical Physics, \\ Moscow State University, 119899, Moscow, Russia
}

(Dated: July 6, 2018)

\begin{abstract}
We study the behavior of quark and diquark condensates at finite Unruh temperature as seen by an accelerated observer. The gap equations for these condensates have been obtained with consideration of a finite chemical potential. Critical values of the acceleration for the restoration of chiral and color symmetries have been estimated.

PACS numbers: 04.62.+v, 11.10.Wx, 11.30.Rd.
\end{abstract}

\section{INTRODUCTION}

It is well known that, according to the Hawking-Unruh effect $[1,2]$, an observer accelerated uniformly in the QCD vacuum behaves as if he were in a thermal bath at the Unruh temperature $T_{U}=a / 2 \pi$ ( $a$ is an acceleration constant). For interacting field theories this is demonstrated by the fact that Euclidean Green's functions written in terms of the Rindler coordinates are periodic in time, and therefore they may be interpreted as thermal. Several problems have been studied in relation to the Hawking-Unruh effect: see, for instance, [3] concerning the discussion of a uniformly accelerated oscillator, or [4] with the study of pair creation by a homogeneous electric field from the point of view of an accelerated observer. At the same time, in a recent paper [5], it was argued that the phase transition from a color glass condensate to a quark gluon plasma through the mechanism of the Hawking-Unruh thermalization can become experimentally observable in relativistic heavy ion collisions.

It was proposed more than twenty years ago $[6,7,8]$ that at high baryon densities a colored diquark condensate $<q q>$ might appear. In analogy with the ordinary superconductivity, this effect was called color superconductivity (CSC). In particular, the CSC phenomenon was investigated in the framework of the one-gluon exchange approximation in QCD [9], where the colored Cooper pair formation is predicted selfconsistently at extremely high values of the chemical potential $\mu \gtrsim 10^{8} \mathrm{MeV}$ [10]. Unfortunately, such baryon densities are not observable in nature and not accessible in experiments (the typical densities inside the neutron stars or in the future heavy ion experiments correspond to $\mu \sim 500 \mathrm{MeV}$ ). In order to study the problem at lower values of $\mu$, various effective theories for low energy QCD, such as the instanton model [11] and the Nambu-Jona-Lasinio (NJL) model[12] can be employed.

It is well known that effective field theories with four-fermion interaction (the so-called Nambu - Jona-Lasinio (NJL) models), which incorporate the phenomenon of dynamical chiral symmetry breaking, are quite useful in describing lowenergy hadronic processes (see e.g. [13,14] and references therein). Since the NJL model displays the same symmetries as QCD, it can be successfully used for simulating some of the QCD ground state properties under the influence of external conditions such as temperature, baryonic chemical potential, or even curved spacetime $[14,15,16,17,18]$. In particular, the role of the NJL approach increases, when detailed numerical lattice calculations are not yet admissible in QCD with nonzero baryon density and in the presence of external gauge fields [19, 20, 21].

The possibility for the existency of the CSC phase in the region of moderate densities was recently proved (see, e.g., the papers $[11,17,22,23]$ as well as the review articles [24] and references therein). In these papers it was shown that the diquark condensate $\langle q q>$ can appear already at a rather moderate baryon density $(\mu \sim 400 \mathrm{MeV})$. The conditions favorable for this condensate to be formed can possibly exist in the cores of cold neutron stars. Since quark Cooper pairing occurs in the color anti-triplet channel, a nonzero value of $\langle q q>$ means that, apart from the electromagnetic $U(1)$ symmetry, the color $S U_{c}(3)$ symmetry should be spontaneously broken inside the CSC phase as well. In the framework of NJL models the CSC phase formation has generally been considered as a dynamical competition between diquark $\langle q q>$ and usual quark-antiquark condensation $<\bar{q} q>$.

Recently, the dynamical chiral symmetry breaking and its restoration for a uniformly accelerated observer due to the thermalization effect of acceleration was studied in [25] at zero chemical potential. Further investigations of the possible influence of the Unruh temperature on the phase transitions in dense quark matter with a finite chemical potential, and especially on the restoration of the broken color symmetry in CSC is thus especially interesting. Related problems have also been studied for chiral symmetry breaking in curved spacetime [16,26], which may be useful for the investigation of compact stars, where the gravitational field is strong and its effect cannot be neglected. Obviously, the results of these studies might have some relevance to the physics of black holes, whose surface gravity causes the finite temperature effects (the Hawking effect). The Rindler metric may be regarded here as an approximation of the 
situation near the event horizon of a black hole. Thus, the study of phase transitions in quark matter with quark and diquark condensates under the influence of acceleration or, equivalently, strong gravitational fields is of great interest.

In this paper, we study quark and diquark condensates as functions of the Unruh temperature and finite chemical potential by using a NJL-type model formulated in Rindler coordinates. As our main result, from the gap equations for these condensates written in Rindler coordinates, the critical values of acceleration (the critical Hawking-Unruh temperatures) for the restoration of the broken chiral and color symmetries were obtained. The results exactly coincide with the usual temperature restoration of these symmetries, when the corresponding relation between the acceleration and the Unruh temperature is taken into account.

\section{NAMBU-JONA-LASINIO MODEL IN RINDLER COORDINATES}

The required effective NJL field theory in Rindler coordinates will be obtained by a suitable transformation of a NJL-type model in flat spacetime with Minkowski coordinates $\left(x^{0}, x^{1}, \vec{x}_{\perp}\right)$. For this aim, let us first quote some useful definitions and formulas. The physics for an accelerated observer can be described by transforming to the Rindler coordinates $\left(\eta, \rho, \vec{x}_{\perp}\right)$ by means of the following coordinate transformation:

$$
x^{0}=\rho \sinh a \eta, \quad x^{1}=\rho \cosh a \eta, \quad x^{i}=x^{i} \quad(i=2,3),
$$

defined on the right Rindler wedge,

$$
0<\rho<+\infty, \quad-\infty<\eta<+\infty
$$

and on the left Rindler wedge,

$$
-\infty<\rho<0, \quad-\infty<\eta<+\infty
$$

where $\eta$ is the time variable in Rindler coordinates.

The gamma-matrices $\gamma_{\mu}$, the metric $g_{\mu \nu}$ and the vierbein $e_{\hat{a}}^{\mu}$, as well as the definitions of the covariant derivative $\nabla_{\nu}$ and spin connection $\omega_{\nu}^{\hat{a} \hat{b}}$ are given by the following relations [27]:

$$
\begin{aligned}
& \left\{\gamma_{\mu}(x), \gamma_{\nu}(x)\right\}=2 g_{\mu \nu}(x), \quad\left\{\gamma_{\hat{a}}, \gamma_{\hat{b}}\right\}=2 \eta_{\hat{a} \hat{b}}, \quad \eta_{\hat{a} \hat{b}}=\operatorname{diag}(1,-1,-1,-1), \\
& g_{\mu \nu} g^{\nu \rho}=\delta_{\mu}^{\rho}, \quad g^{\mu \nu}(x)=e_{\hat{a}}^{\mu}(x) e^{\nu \hat{a}}(x), \quad \gamma_{\mu}(x)=e_{\mu}^{\hat{a}}(x) \gamma_{\hat{a}} . \\
& \nabla_{\nu} \equiv \partial_{\nu}+\frac{1}{2} \sigma_{\hat{a} \hat{b}} \omega_{\nu}^{\hat{a} \hat{b}}, \quad \sigma_{\hat{a} \hat{b}} \equiv \frac{1}{4}\left[\gamma_{\hat{a}}, \gamma_{\hat{b}}\right], \\
& \omega_{\mu}^{\hat{a} \hat{b}} \equiv \frac{1}{2} e^{\hat{a} \lambda} e^{\hat{b} \rho}\left[C_{\lambda \rho \mu}-C_{\rho \lambda \mu}-C_{\mu \lambda \rho}\right], \quad C_{\lambda \rho \mu} \equiv e_{\lambda}^{\hat{a}} \partial_{[\rho} e_{\mu] \hat{a}}
\end{aligned}
$$

Here, the index $\hat{a}$ refers to the flat tangent space defined by the vierbein at spacetime point $x$, and the $\gamma^{\hat{a}}(a=0,1,2,3)$ are the usual Dirac gamma-matrices of Minkowski spacetime.

The line element

$$
d s^{2}=\eta_{\hat{a} \hat{b}} e_{\mu}^{\hat{a}} e_{\nu}^{\hat{b}} d x^{\mu} d x^{\nu}
$$

in these coordinates with the vierbeins

$$
\mathrm{e}_{0}^{\hat{0}}=a \rho, \quad e_{1}^{\hat{1}}=\ldots=1
$$

is given by the relation

$$
d s^{2}=a^{2} \rho^{2} d \eta^{2}-d \rho^{2}-d \vec{x}_{\perp}^{2}
$$

with the metric tensor

$$
g_{\mu \nu}=\left(a^{2} \rho^{2},-1,-1,-1\right) .
$$

In what follows, we shall limit our consideration to the right Rindler wedge. An observer at fixed $\rho, \vec{x}_{\perp}$ measures a proper time $d \tau=a \rho d \eta$ and has a proper acceleration $1 / \rho$. The observer at $\rho=1 / a$ measures $d \tau=d \eta$ and has a proper acceleration $a$. The world line of the observer in Rindler coordinates is thus given as

$$
\eta(\tau)=\tau, \quad \rho(\tau)=1 / a, \quad \vec{x}_{\perp}(\tau)=\text { const }
$$


For an accelerated observer moving with constant acceleration according to (4), the gamma matrices in Rindler coordinates are obtained by the definition given in (1)

$$
\gamma_{0}=a^{2} \rho^{2} \gamma^{0}=a \rho \gamma_{\hat{0}}, \quad \gamma_{\mu}=g_{\mu \nu} \gamma^{\nu}
$$

and hence

$$
\gamma^{0}(x)=\frac{1}{a \rho} \gamma^{\hat{0}}, \quad \gamma^{1}(x)=\gamma^{\hat{1}}, \quad \gamma^{2}(x)=\gamma^{\hat{2}}, \quad \gamma^{3}(x)=\gamma^{\hat{3}} .
$$

Using the definition given in (2) for computing the spin connection, one finds the components of the covariant derivatives in Rindler coordinates:

$$
\nabla_{0}=\partial_{\eta}+\frac{a}{2} \gamma_{\hat{0}} \gamma_{\hat{1}}, \quad \nabla_{1}=\partial_{\rho}, \quad \nabla_{2}=\partial_{2}, \quad \nabla_{3}=\partial_{3} .
$$

After these intoductions, let us consider the following four-quark model of up- and down-quarks with $(\bar{q} q)$ and $(q q)$ interactions in the color group $S U_{c}\left(N_{c}\right)$ given by the generalization of the corresponding Lagrangian for the Nambu-Jona-Lasinio model in flat space [21, 23], [32]

$$
\begin{aligned}
\mathcal{L} & =\bar{q}(x)\left[\mathrm{i} \gamma^{\nu}(x) \nabla_{\nu}+\mu \gamma^{0}(x)\right] q+\frac{G_{1}}{2 N_{c}}\left[(\bar{q}(x) q(x))^{2}+\left(\bar{q}(x) \mathrm{i} \gamma^{5} \vec{\tau} q(x)\right)^{2}\right]+ \\
& +\frac{G_{2}}{N_{c}} \sum_{b}\left[\mathrm{i} \bar{q}(x)_{c} \varepsilon\left(\mathrm{i} \lambda_{a s}^{b}\right) \gamma^{5} q(x)\right]\left[\mathrm{i} \bar{q}(x) \varepsilon\left(\mathrm{i} \lambda_{a s}^{b}\right) \gamma^{5} q_{c}(x)\right] .
\end{aligned}
$$

In (8) $\mu$ is the quark chemical potential, $q_{c}=C \bar{q}^{t}, \bar{q}_{c}=q^{t} C$ are charge-conjugated spinors, and $C=\mathrm{i} \gamma^{2} \gamma^{0}$ is the charge conjugation matrix ( $t$ denotes the transposition operation). It is necessary to note that in order to obtain realistic estimates for masses of vector/axial-vector mesons and diquarks in extended NJL-type of models [13, 29], we have to allow for independent coupling constants $G_{1}, G_{2}$, rather than to consider them related by a Fierz transformation of a current-current interaction via gluon exchange. In what follows we assume $N_{c}=3$ and replace the antisymmetric color matrices $\lambda_{\text {as }}^{b}$ (with a factor i) by the antisymmetric $\epsilon^{b}$ operator. The quark field $q \equiv q_{i \alpha}$ is a flavor doublet and color triplet as well as a four-component Dirac spinor, where $i=1,2 ; \alpha=1,2,3$. (Latin and Greek indices refer to flavor and color indices, respectively; spinor indices are omitted.) Furthermore, $\vec{\tau} \equiv\left(\tau^{1}, \tau^{2}, \tau^{3}\right)$ are Pauli matrices in the flavor space; $(\varepsilon)^{i k} \equiv \varepsilon^{i k},\left(\epsilon^{b}\right)^{\alpha \beta} \equiv \epsilon^{\alpha \beta b}$ are totally antisymmetric tensors in the flavor and color spaces, respectively. Clearly, the Lagrangian (8) is invariant under the chiral $S U(2)_{L} \times S U(2)_{R}$ and color $S U_{c}(3)$ groups.

Next, by applying the usual bosonization procedure, we obtain the linearized version of the model (8) with collective bosonic fields

$$
\begin{aligned}
\tilde{\mathcal{L}} & =\bar{q}\left[\mathrm{i} \gamma^{\nu} \nabla_{\nu}+\mu \gamma^{0}\right] q-\bar{q}\left(\sigma+\mathrm{i} \gamma^{5} \vec{\tau} \vec{\pi}\right) q-\frac{3}{2 G_{1}}\left(\sigma^{2}+\vec{\pi}^{2}\right)- \\
& -\frac{3}{G_{2}} \Delta^{* b} \Delta^{b}-\Delta^{* b}\left[\mathrm{i} q^{t} C \varepsilon \epsilon^{b} \gamma^{5} q\right]-\Delta^{b}\left[\mathrm{i} \bar{q} \varepsilon \epsilon^{b} \gamma^{5} C \bar{q}^{t}\right] .
\end{aligned}
$$

The Lagrangians (8) and (9) are equivalent, as can be seen by using the equations of motion for bosonic fields, from which it follows that

$$
\Delta^{b} \sim \mathrm{i} q^{t} C \varepsilon \epsilon^{b} \gamma^{5} q, \quad \sigma \sim \bar{q} q, \quad \vec{\pi} \sim \mathrm{i} \bar{q} \gamma^{5} \vec{\tau} q .
$$

Clearly, the $\sigma$ and $\vec{\pi}$ fields are color singlets. Besides, the (bosonic) diquark field $\Delta^{b}$ is a color antitriplet and a (isoscalar) singlet under the chiral $S U(2)_{L} \times S U(2)_{R}$ group. Note further that the $\sigma, \Delta^{b}$, are scalars, but the $\vec{\pi}$ are pseudo-scalar fields. Hence, if $\sigma \neq 0$, then chiral symmetry of the model is spontaneously broken, whereas $\Delta^{b} \neq 0$ indicates the dynamical breaking of both the color and electromagnetic symmetries of the theory. In what follows, for our purpose of investigating the ground state of the system, we may suppose that all boson fields do not depend on space-time.

In the one-loop approximation, the partition function can be written as follows:

$$
Z=\exp \left(\mathrm{i} S_{\mathrm{eff}}\left(\sigma, \vec{\pi}, \Delta^{b}, \Delta^{* b}\right)\right)
$$

where

$$
S_{\mathrm{eff}}\left(\sigma, \vec{\pi}, \Delta^{b}, \Delta^{* b}\right)=-N_{c} \int d^{4} x \sqrt{-g}\left[\frac{\sigma^{2}+\vec{\pi}^{2}}{2 G_{1}}+\frac{\Delta^{b} \Delta^{* b}}{G_{2}}\right]+S_{q}
$$


is the effective action for the boson fields. The quark contribution to the effective action, $S_{q}$, is expressed through the path integral over quark fields

$$
Z_{q}=\exp \left(\mathrm{i} S_{q}\right)=N^{\prime} \int d \bar{q} d q \exp \left(\mathrm{i} \int d^{4} x \sqrt{-g}\left[\bar{q} D q+\bar{q} \mathcal{M} \bar{q}^{t}+q^{t} \overline{\mathcal{M}} q\right]\right) .
$$

where $N^{\prime}$ is a normalization constant.

In (12) we have used the following notations

$$
D=\mathrm{i} \gamma^{\mu} \nabla_{\mu}-\sigma-\mathrm{i} \gamma^{5} \vec{\pi} \vec{\tau}+\mu \gamma^{0}, \quad \overline{\mathcal{M}}=-\mathrm{i} \Delta^{* b} C \varepsilon \epsilon^{b} \gamma^{5}, \quad \mathcal{M}=-\mathrm{i} \Delta^{b} \varepsilon \epsilon^{b} \gamma^{5} C,
$$

where $D$ is an operator in the coordinate, spinor and flavor spaces, whereas $\mathcal{M}$ and $\overline{\mathcal{M}}$ are operators in the color space as well. Next, assume that in the ground state of our model $\left\langle\Delta^{1}\right\rangle=\left\langle\Delta^{2}\right\rangle=\langle\vec{\pi}\rangle=0$ and $\langle\sigma\rangle,\left\langle\Delta^{3}\right\rangle \neq 0[33]$. Obviously, the residual color symmetry group of such a vacuum is $S U_{c}(2)$ whose generators are the first three generators of the initial $S U_{c}(3)$.

Due to our assumption on the vacuum structure, we put $\Delta^{1,2} \rightarrow\left\langle\Delta^{1,2}\right\rangle \equiv 0, \vec{\pi} \rightarrow\langle\vec{\pi}\rangle=0,\left\langle\Delta^{3}\right\rangle \equiv \Delta,\langle\sigma\rangle \equiv \sigma$. One can easily see that the functional integral in (12) can be factorized

$$
\begin{gathered}
Z_{q}=\exp \left(\mathrm{i} S_{q}\right)=N^{\prime} \int d \bar{q}_{3} d q_{3} \exp \left(\mathrm{i} \int d^{4} x \sqrt{-g} \bar{q}_{3} \tilde{D} q_{3}\right) \\
\times \int d \bar{Q} d Q \exp \left(\mathrm{i} \int d^{4} x \sqrt{-g}\left[\bar{Q} \tilde{D} Q+\bar{Q} M \bar{Q}^{t}+Q^{t} \bar{M} Q\right]\right),
\end{gathered}
$$

where $q_{3}$ is the quark field of color 3 , and $Q \equiv\left(q_{1}, q_{2}\right)^{t}$ is the doublet, composed from quark fields of the colors 1,2 . Moreover,

$$
\tilde{D}=\left.D\right|_{\vec{\pi}=0}, \quad \bar{M}=-i \Delta^{*} C \varepsilon \tilde{\epsilon} \gamma^{5}, \quad M=-i \Delta \varepsilon \tilde{\epsilon} \gamma^{5} C
$$

In $(15), \tilde{\epsilon}$ is the matrix in the two-dimensional color subspace, corresponding to the $S U_{c}(2)$ group:

$$
\tilde{\epsilon}=\left(\begin{array}{cc}
0 & 1 \\
-1 & 0
\end{array}\right)
$$

Clearly, the integration over $q_{3}$ in (14) yields Det $\tilde{D}$.

Defining $\Psi^{t}=\left(Q^{t}, \bar{Q}\right)$ and introducing the matrix-valued operator

$$
Z=\left(\begin{array}{cc}
2 \bar{M}, & -\tilde{D}^{t} \\
\tilde{D}, & 2 M
\end{array}\right)
$$

the Gaussian integral over $\bar{Q}$ and $Q$ in (14) can be rewritten in compact matrix notation and be evaluated as

$$
\int d \Psi \mathrm{e}^{\frac{\mathrm{i}}{2} \int d^{4} x \sqrt{-g} \Psi^{t} Z \Psi}=\sqrt{\operatorname{Det} Z}
$$

Then, by using in (16) the general formula

$$
\operatorname{Det}\left(\begin{array}{cc}
U & , \\
\bar{V} & \bar{U}
\end{array}\right)=\operatorname{Det}\left[-\bar{V} V+\bar{V} U \bar{V}^{-1} \bar{U}\right]=\operatorname{Det}\left[\bar{U} U-\bar{U} V \bar{U}^{-1} \bar{V}\right],
$$

one obtains the result:

$$
\begin{gathered}
\exp \left(\mathrm{i} S_{q}\right)=N^{\prime} \operatorname{Det} \tilde{D} \operatorname{Det}^{1 / 2}\left[4 M \bar{M}+M \tilde{D}^{t} M^{-1} \tilde{D}\right] \\
=N^{\prime} \operatorname{Det}\left(\mathrm{i} \gamma^{\nu} \nabla_{\nu}-\sigma+\mu \gamma^{0}\right) \operatorname{Det}^{1 / 2}\left[4|\Delta|^{2}+\left(-\mathrm{i} \gamma^{\nu} \nabla_{\nu}-\sigma+\mu \gamma^{0}\right)\left(\mathrm{i} \gamma^{\mu} \nabla_{\mu}-\sigma+\mu \gamma^{0}\right)\right] .
\end{gathered}
$$

Recall that the first Det-operation in (17) acts only in the flavor, coordinate and spinor spaces, whereas the second Det-operation acts in the two-dimensional color subspace, as well. The quark contribution to the effective action

$$
S_{q}=-\mathrm{i} \operatorname{Tr} \log \left(\mathrm{i} \gamma^{\nu} \nabla_{\nu}-\sigma+\mu \gamma^{0}\right)-\frac{\mathrm{i}}{2} \operatorname{Tr} \log \left[4|\Delta|^{2}+\left(-\mathrm{i} \gamma^{\nu} \nabla_{\nu}-\sigma+\mu \gamma^{0}\right)\left(\mathrm{i} \gamma^{\mu} \nabla_{\mu}-\sigma+\mu \gamma^{0}\right)\right]
$$


can be written in the form

$$
S_{q}=S_{q 1}+S_{q 2}=-\frac{\mathrm{i}}{2}\left[\operatorname{tr} \log B_{1}^{2}+2 \operatorname{tr} \log B_{2}^{2}\right]
$$

where we have summed over colors (leading to the factor 2 in the second term; the tr -operation does not include color indices any more) and

$$
\begin{gathered}
B_{1}^{2}=\left(-\mathrm{i} \gamma^{\nu} \nabla_{\nu}-\sigma-\mu \gamma^{0}\right)\left(\mathrm{i} \gamma^{\mu} \nabla_{\mu}-\sigma+\mu \gamma^{0}\right) \\
B_{2}^{2}=4|\Delta|^{2}+\left(-\mathrm{i} \gamma^{\nu} \nabla_{\nu}-\sigma+\mu \gamma^{0}\right)\left(\mathrm{i} \gamma^{\mu} \nabla_{\mu}-\sigma+\mu \gamma^{0}\right)
\end{gathered}
$$

Here the product of the relevant operators appearing in (19) can be represented in Rindler coordinates as

$$
B^{2} \equiv\left(-\mathrm{i} \gamma^{\nu} \nabla_{\nu}-\sigma\right)\left(\mathrm{i} \gamma^{\mu} \nabla_{\mu}-\sigma\right)=\frac{1}{\rho^{2}}\left[\frac{1}{a} \partial_{\eta}+\frac{1}{2} \gamma_{\hat{0}} \gamma_{\hat{1}}\right]^{2}-\left[\frac{\partial^{2}}{\partial \rho^{2}}+\frac{1}{\rho} \frac{\partial}{\partial \rho}-(\vec{\gamma} \vec{\nabla})_{\perp}^{2}-\sigma^{2}\right]
$$

For the following, it is convenient to represent the operators $B_{1}^{2}, B_{2}^{2}$ in the basis of the solutions of the squared Dirac equation

$$
B^{2} \Psi_{\vec{k}_{\perp}, j}\left(\eta, \vec{x}_{\perp}, \rho\right)=0
$$

The solutions can be sought in the form

$$
\Psi_{\vec{k}_{\perp, j}}\left(\eta, \vec{x}_{\perp}, \rho\right)=\mathrm{e}^{-\mathrm{i} a j \eta} \mathrm{e}^{\mathrm{i} \vec{k}_{\perp} \vec{x}_{\perp}} \psi_{j}(\rho)
$$

and hence, with consideration of $(20)$, the function $\psi_{j}(\rho)$ is the solution of the second order Bessel differential equation forming the basis of the Rindler modes (see, e.g., [1])

$$
\left(\rho^{2} \frac{d^{2}}{d \rho^{2}}+\rho \frac{d}{d \rho}-m^{2} \rho^{2}+E_{j}^{2}\right) \psi_{j}(\rho)=0
$$

where for the Rindler modes in the fermion sector, we have to take

$$
m^{2}=\vec{k}_{\perp}^{2}+\sigma^{2}, E_{j}^{2}=\left(j \pm \frac{\mathrm{i}}{2}\right)^{2}, 0<j<+\infty .
$$

Two independent solutions of equations $(21),(23)$ can be obtained by using the projection operator $P_{ \pm}=\frac{1}{2}\left(1 \pm \gamma_{\hat{0}} \gamma_{\hat{1}}\right):$

$$
\psi_{j}^{(+)}=P_{+} \psi_{j}, \quad \psi_{j}^{(-)}=P_{-} \psi_{j}
$$

Then the normalized solutions of (23) look as follows:

$$
\psi_{j}^{(-)}(\rho)=\frac{\sqrt{(-2 \mathrm{i} j-1) \cosh \pi j}}{\pi} K_{\mathrm{i} j+\frac{1}{2}}(m \rho), \quad \psi_{j}^{(+)}(\rho)=\frac{\sqrt{(+2 \mathrm{i} j-1) \cosh \pi j}}{\pi} K_{\mathrm{i} j-\frac{1}{2}}(m \rho),
$$

where $K_{\nu}$ is the Macdonald function (modified Bessel function).

These solutions $<\rho \mid \vec{k}_{\perp}, j, \pm>=\psi_{\vec{k}_{\perp, j}}^{( \pm)}(\rho)$, for which we shall use the shorthand notation $\psi_{j}^{( \pm)}(\rho) \equiv \psi_{\vec{k}_{\perp, j}}^{( \pm)}(\rho)$, form a complete set of functions with the orthonormalization condition [28]

$$
\int_{0}^{\infty} \frac{d \rho}{\rho} \psi_{j}^{(\mp)}(\rho) \psi_{j^{\prime}}^{(\mp)}(\rho)=\frac{1}{\pi^{2}} \int_{0}^{\infty} \frac{d \rho}{\rho} \sqrt{(\mp 2 \mathrm{i} j-1) \cosh \pi j\left(\mp 2 \mathrm{i} j^{\prime}-1\right) \cosh \pi j^{\prime}} K_{\mathrm{i} j \pm \frac{1}{2}}(m \rho) K_{\mathrm{i} j^{\prime} \pm \frac{1}{2}}(m \rho)=\delta\left(j-j^{\prime}\right) .
$$

Taking the above formulas and the product of operators (20) into account, we obtain

$$
B_{2}^{2}=4|\Delta|^{2}+\frac{1}{\rho^{2}}\left[\frac{1}{a} \partial_{\eta}+\frac{1}{2} \gamma_{\hat{0}} \gamma_{\hat{1}}\right]^{2}-\left[\frac{\partial^{2}}{\partial \rho^{2}}+\frac{1}{\rho} \frac{\partial}{\partial \rho}-(\vec{\gamma} \vec{\nabla})_{\perp}^{2}-\sigma^{2}\right]+\left(\frac{\mu}{a}\right)^{2} \frac{1}{\rho^{2}}-2 \frac{\mu}{a \rho}\left[\gamma_{\hat{0}} \sigma-\mathrm{i} \gamma_{\hat{0}}(\vec{\gamma} \vec{\nabla})\right] .
$$


For our further calculations, we also have to transform, in the same way, the product of operators in $B_{1}^{2}$ :

$$
B_{1}^{2}=\frac{1}{\rho^{2}}\left[\frac{1}{a} \partial_{\eta}+\frac{1}{2} \gamma_{\hat{0}} \gamma_{\hat{1}}-\mathrm{i} \frac{\mu}{a}\right]^{2}-\left[\frac{\partial^{2}}{\partial \rho^{2}}+\frac{1}{\rho} \frac{\partial}{\partial \rho}-(\vec{\gamma} \vec{\nabla})_{\perp}^{2}-\sigma^{2}\right] .
$$

In order to find nonvanishing condensates $\langle\sigma\rangle$ and $\left\langle\Delta^{3}\right\rangle$, we should calculate the effective potential, whose global minimum point provides us with these quantities. By definition of the effective potential we have

$$
V_{\mathrm{eff}}=-\frac{S_{\mathrm{eff}}}{\int d^{D} x \sqrt{-g}}
$$

In this case, according to (29) we obtain

$$
V_{\mathrm{eff}}=\frac{3 \sigma^{2}}{2 G_{1}}+\frac{3 \Delta^{b} \Delta^{* b}}{G_{2}}-\frac{S_{q}}{\int d^{4} x \sqrt{-g}}
$$

The gap equations correspond to the stationarity condition:

$$
\frac{\partial V_{\mathrm{eff}}}{\partial \Delta_{0}^{3 *}}=0, \frac{\partial V_{\mathrm{eff}}}{\partial \sigma}=0
$$

The gap equations (31) are obtained by differentiating the logarithm in (18) with respect to $\Delta^{3 *}$ and $\sigma$. Moreover, by taking into account that the position of the accelerated observer is defined in (4), we can put $\rho=1 / a$.

\section{CHIRAL SYMMETRY BREAKING}

First, let us consider a simpler problem, chiral symmetry breaking, already discussed in [25] for the case of a vanishing chemical potential, and described qualitatively in the Introduction. In this Section, unlike in [25], a nonzero chemical potential $\mu$ will be taken into account.

Let us first assume that $\Delta=0$ and $\mu=0$. Then according to (18), (20),

$$
S_{q}=-\frac{3}{2} \mathrm{i} \operatorname{tr} \log B^{2}
$$

and the gap equation looks like

$$
\sigma=-\frac{\mathrm{i} G_{1} \sigma}{\int d^{4} x \sqrt{-g}} \operatorname{tr} \frac{1}{B^{2}}
$$

Now we go over to the momentum representation by replacing

$$
\mathrm{i} \frac{\partial}{\partial \eta} \rightarrow k_{0},-\mathrm{i} \vec{\nabla}_{\perp} \rightarrow \vec{k}_{\perp}
$$

and obtain

$$
\sigma=-\mathrm{i} G_{1} \sigma N_{f} \int \frac{d k_{0}}{2 \pi} \int \frac{d^{2} k_{\perp}}{(2 \pi)^{2}} \int_{0}^{\infty} \frac{d \rho}{\rho}<\vec{k}_{\perp}, k_{0}, \rho\left|\frac{1}{B^{2}}\right| \vec{k}_{\perp}, k_{0}, \rho>\left.\right|_{\rho=a^{-1}}
$$

where $N_{f}=2$ is the number of flavors in our problem. With the use of the completeness relation (25) of the Rindler basis $<\rho \mid \vec{k}_{\perp}, j, \pm>=\psi_{\vec{k}_{\perp}, j}^{( \pm)}(\rho)$, let us go over to this basis in the variable $\rho$, so that

$$
\rho^{2} \frac{d^{2}}{d \rho^{2}}+\rho \frac{d}{d \rho}-m^{2} \rho^{2} \rightarrow-\left(j \pm \frac{\mathrm{i}}{2}\right)^{2} .
$$

Next, we are going to an imaginary time coordinate, i.e., to the Euclidean spacetime in order to consider the thermal effect of acceleration $[2,30]$. The Euclidean Rindler spacetime has a singularity at $\rho=0$, therefore we have to choose the period of the imaginary time as $2 \pi / a[27]$. The Euclidean formalism in Rindler coordinates with a definite period 
$\beta=2 \pi / a$ of imaginary time coincides with the finite-temperature imaginary time Matsubara formalism, which is realized by the following substitutions in our equations [31]:

$$
\int \frac{d k_{0}}{2 \pi} \rightarrow \sum_{n} \frac{1}{\beta}, \quad k_{0} \rightarrow \mathrm{i} \omega_{n}
$$

where $\omega_{n}$ is the discrete fermion frequency defined by $\omega_{n}=(2 n+1) \pi / \beta(n=0, \pm 1, \pm 2, \cdots)$.

After this, we finally obtain

$$
\sigma=-G_{1} \sigma N_{f} \sum_{n} \frac{1}{2 \pi} \int \frac{d^{2} k_{\perp}}{(2 \pi)^{2}} \sum_{ \pm} \int_{0}^{\infty} d j \frac{\left[\frac{1}{\pi} \sqrt{(\mp 2 \mathrm{i} j-1) \cosh \pi j} K_{\mathrm{i} j \pm \frac{1}{2}}\left(m a^{-1}\right)\right]^{2}}{\left(\frac{\mathrm{i} \omega_{n}}{a}-j \pm \mathrm{i}\right)\left(\frac{\mathrm{i} \omega_{n}}{a}+j\right)}
$$

Now, consider the case of a nonzero chemical potential $\mu$. Take into consideration that under the operation of charge conjugation performed in the above $B_{1,2}^{2}$ operators one obtains $\mu \rightarrow-\mu$. Hence we should take both signs, $\pm \mu$, into consideration (this is equivalent to considering also for another branch of the solution of the Rindler equation with $j \rightarrow-j)$. Therefore

$$
S_{q 1}=-\frac{\mathrm{i}}{2} \operatorname{tr} \log B_{1}^{2} \rightarrow-\frac{\mathrm{i}}{2} \operatorname{tr} \log B_{1+}^{2} B_{1-}^{2}
$$

where

$$
B_{1 \zeta}^{2}=\frac{1}{\rho^{2}}\left[\frac{1}{a} \partial_{\eta}+\frac{1}{2} \gamma_{\hat{0}} \gamma_{\hat{1}}-\mathrm{i} \zeta \frac{\mu}{a}\right]^{2}-\left[\frac{\partial^{2}}{\partial \rho^{2}}+\frac{1}{\rho} \frac{\partial}{\partial \rho}-(\vec{\gamma} \vec{\nabla})_{\perp}^{2}-\sigma^{2}\right] \quad \text { with } \quad \zeta= \pm 1 .
$$

Then, taking the Rindler basis (22), (25) into consideration, in the Euclidean spacetime with Matsubara frequencies the above operator takes the form

$$
B_{1 \zeta}^{2}=\frac{1}{\rho^{2}}\left[\frac{\omega_{n}}{a} \pm \frac{1}{2}-\mathrm{i} \zeta \frac{\mu}{a}\right]^{2}+\frac{1}{\rho^{2}}\left[j \mp \frac{\mathrm{i}}{2}\right]^{2}
$$

We can write the identity

$$
\begin{gathered}
B_{1+}^{2} B_{1-}^{2}=\left(\frac{1}{\rho^{2}}\left[j \mp \frac{\mathrm{i}}{2}\right]^{2}+\frac{1}{\rho^{2}}\left[\frac{\omega_{n}}{a} \pm \frac{1}{2}-\mathrm{i} \frac{\mu}{a}\right]^{2}\right) \cdot\left(\frac{1}{\rho^{2}}\left[j \mp \frac{\mathrm{i}}{2}\right]^{2}+\frac{1}{\rho^{2}}\left[\frac{\omega_{n}}{a} \pm \frac{1}{2}+\mathrm{i} \frac{\mu}{a}\right]^{2}\right) \\
=\left(\frac{1}{\rho^{2}}\left[j \mp \frac{\mathrm{i}}{2}+\frac{\mu}{a}\right]^{2}+\frac{1}{\rho^{2}}\left[\frac{\omega_{n}}{a} \pm \frac{1}{2}\right]^{2}\right) \cdot\left(\frac{1}{\rho^{2}}\left[j \mp \frac{\mathrm{i}}{2}-\frac{\mu}{a}\right]^{2}+\frac{1}{\rho^{2}}\left[\frac{\omega_{n}}{a} \pm \frac{1}{2}\right]^{2}\right) .
\end{gathered}
$$

From the Bessel equation (23) we have

$$
<\vec{k}_{\perp}, j, \pm\left|-2 \sigma d \sigma \rho^{2}+2 E_{j} d j\right| \vec{k}_{\perp}, j, \pm>=0
$$

and hence in the case of finite $\mu$ we obtain instead of (33)

$$
\sigma=-G_{1} \sigma N_{f} \sum_{\zeta} \sum_{n} \frac{1}{2 \pi} \int \frac{d^{2} k_{\perp}}{(2 \pi)^{2}} \sum_{ \pm} \int_{0}^{\infty} d j \frac{j \mp \frac{\mathrm{i}}{2}-\zeta \frac{\mu}{a}}{j \mp \frac{\mathrm{i}}{2}} \cdot \frac{\left[\frac{1}{\pi} \sqrt{(\mp 2 \mathrm{i} j-1) \cosh \pi j} K_{\mathrm{i} j \pm \frac{1}{2}}\left(m a^{-1}\right)\right]^{2}}{\left(\frac{\mathrm{i} \omega_{n}}{a}-\left(j-\zeta \frac{\mu}{a}\right) \pm \mathrm{i}\right)\left(\frac{\mathrm{i} \omega_{n}}{a}+j-\zeta \frac{\mu}{a}\right)} .
$$

Now by summing over Matsubara frequencies $\omega_{n}=a\left(n+\frac{1}{2}\right)$ with the help of the formula

$$
\sum_{n} \frac{1}{\left(\frac{\mathrm{i} \omega_{n}}{a}-\left(j-\zeta \frac{\mu}{a}\right) \pm \mathrm{i}\right)\left(\frac{\mathrm{i} \omega_{n}}{a}+j-\zeta \frac{\mu}{a}\right)}=\mp \frac{2 \pi \mathrm{i}}{1 \pm 2 \mathrm{i}\left(j-\zeta \frac{\mu}{a}\right)} \tanh \pi\left(j-\zeta \frac{\mu}{a}\right),
$$

the final result is obtained

$$
\sigma=-\mathrm{i} G_{1} \sigma N_{f} \sum_{\zeta} \sum_{ \pm} \int \frac{d^{2} k_{\perp}}{(2 \pi)^{2}} \int_{0}^{\infty} d j \cosh \pi j \frac{\tanh \pi\left(j-\zeta \frac{\mu}{a}\right)}{\pi^{2}}\left(K_{\mathrm{i} j \pm \frac{1}{2}}\left(m a^{-1}\right)\right)^{2} .
$$


For illustrations, let us estimate the critical acceleration $a_{c}$, when the quark condensate vanishes. To this end, we put $\sigma=0\left(m \rightarrow k_{\perp}\right)$ and integrate over $\vec{k}_{\perp}$ with the help of the integral

$$
\int_{0}^{\infty} k d k K_{n}\left(k a^{-1}\right)^{2}=\frac{1}{2} a^{2} \frac{n \pi}{\sin n \pi}
$$

The final result looks as follows:

$$
1=\frac{G_{1}}{2 \pi^{2}} N_{f} \sum_{\zeta} \int_{0}^{\infty} d q q \tanh \left(\pi \frac{q-\zeta \mu}{a}\right)
$$

The above equation precisely corresponds to the known expression for the critical curve, obtained for finite temperature and chemical potential (see, e.g. [17]), if the correspondence between the acceleration $a$ and Unruh temperature $T$ is taken into consideration,

$$
\frac{\pi}{a}=\frac{1}{2 T}
$$

Now, recall that the Unruh temperature is given by the relation

$$
T=\frac{a}{2.5 \times 10^{22}\left(\mathrm{~cm} \mathrm{~s}^{-2}\right)} \mathrm{K} .
$$

Let us take the value of the maximum critical temperature on the transition curve for the quark condensate formation $T_{m}=0.169 \mathrm{GeV}$, calculated in [17]. Then, we find for the critical acceleration the following estimate $a_{c}=2 \pi T=$ $2 \pi \times 0.169 \mathrm{GeV}=3.2 \times 10^{35} \mathrm{~cm} / \mathrm{s}^{2}$. This value is an order of magnitude larger than the value found for the case of a vanishing chemical potential in [25].

\section{COLOR SYMMETRY BREAKING AND FORMATION OF A DIQUARK CONDENSATE}

In order to study the minimum in the variable $\Delta$ of the diquark condensate, we may here put $\sigma=0$. Now, we have

$$
S_{q 2}=-\mathrm{i} \operatorname{tr} \log B_{2}^{2}=-\mathrm{i} \operatorname{tr} \log \left[4|\Delta|^{2}+\left(-\mathrm{i} \gamma^{\nu} \nabla_{\nu}+\mu \gamma^{0}\right)\left(\mathrm{i} \gamma^{\mu} \nabla_{\mu}+\mu \gamma^{0}\right)\right]
$$

Then after taking into account charge conjugation

$$
S_{q 2} \rightarrow-\mathrm{i} \operatorname{tr} \log B_{2+}^{2} B_{2-}^{2}
$$

where

$$
B_{2 \zeta}^{2}=4|\Delta|^{2}+\left(-\mathrm{i} \gamma^{\nu} \nabla_{\nu}+\zeta \mu \gamma^{0}\right)\left(\mathrm{i} \gamma^{\mu} \nabla_{\mu}+\zeta \mu \gamma^{0}\right)
$$

The corresponding gap equation now takes the form

$$
\frac{3 \Delta}{G_{2}}=\frac{1}{\int d^{4} x \sqrt{-g}} \frac{\partial S_{q 2}}{\partial \Delta} .
$$

Let us again estimate the value of the critical Unruh temperature and acceleration, at which the broken color symmetry is restored. For this purpose, we now put $\Delta=0$. Then the operators in the above equation can be expanded in the Rindler basis (25), and after going over to the Euclidean spacetime and Matsubara frequencies the gap equation can again be written in the form

$$
\begin{aligned}
1= & \frac{4}{3} G_{2} N_{f} \sum_{n} \sum_{\zeta} \frac{1}{2 \pi} \int \frac{d^{2} k_{\perp}}{(2 \pi)^{2}} \int_{0}^{\infty} d j \\
& \times\left\{\frac{(-2 \mathrm{i} j-1) \cosh \pi j}{\pi^{2}}\left(K_{\mathrm{i} j+\frac{1}{2}}\left(m a^{-1}\right)\right)^{2} \frac{1}{\left(\left(\mathrm{i} \omega_{n}+\zeta \mu\right) / a-j+\mathrm{i}\right)\left(\left(\mathrm{i} \omega_{n}-\zeta \mu\right) / a+j\right)}\right. \\
& \left.\quad+\frac{(2 \mathrm{i} j-1) \cosh \pi j}{\pi^{2}}\left(K_{\mathrm{i} j-\frac{1}{2}}\left(m a^{-1}\right)\right)^{2} \frac{1}{\left(\left(\mathrm{i} \omega_{n}+\zeta \mu\right) / a-j-\mathrm{i}\right)\left(\left(\mathrm{i} \omega_{n}-\mu\right) / a+j\right)}\right\}
\end{aligned}
$$


where now $m^{2}=\vec{k}_{\perp}^{2}$.

Next, we have to sum over the Matsubara frequencies in the above equation with the help of the formula (38). As a result, we obtain

$$
\begin{aligned}
1=- & \frac{4}{3} \frac{G_{2}}{2 \pi} N_{f} \int \frac{d^{2} k_{\perp}}{(2 \pi)^{2}} \int_{0}^{\infty} d j \sum_{\zeta} 2 \mathrm{i} \pi \tanh \pi\left(j-\zeta \frac{\mu}{a}\right) \\
& \times\left\{\frac{(2 \mathrm{i} j+1) \cosh \pi j}{\pi^{2}}\left(K_{\mathrm{i} j+\frac{1}{2}}\left(m a^{-1}\right)\right)^{2} \frac{1}{1+2 \mathrm{i}(j-\zeta \mu / a)}\right. \\
& \left.+\frac{(2 \mathrm{i} j-1) \cosh \pi j}{\pi^{2}}\left(K_{\mathrm{i} j-\frac{1}{2}}\left(m a^{-1}\right)\right)^{2} \frac{1}{1-2 \mathrm{i}(j-\zeta \mu / a)}\right\} .
\end{aligned}
$$

Now, the integration over $k$ can be performed with the use of the integral (40). Then we have

$$
1=-\mathrm{i} \frac{2}{3(2 \pi)^{2}} G_{2} a^{2} N_{f} \sum_{\zeta} \int_{0}^{\infty} d j \tanh \pi(j-\zeta \mu / a)\left[\frac{(1+2 \mathrm{i} j)^{2}}{1+2 \mathrm{i}(j-\zeta \mu / a)}-\frac{(1-2 \mathrm{i} j)^{2}}{1-2 \mathrm{i}(j-\zeta \mu / a)}\right] .
$$

Changing the integration variable as $j \rightarrow q=a j$, and taking into consideration that in the physical region $q / a \gg 1,(q-\mu) / a \gg 1$, the above integral can be approximated by

$$
1=\frac{2}{3} \frac{G_{2}}{\pi^{2}} N_{f}\left[\int_{0}^{\Lambda} d q q^{2} \frac{\tanh \frac{\pi(q+\mu)}{a}}{q+\mu}+\int_{\mu}^{\Lambda} d q q^{2} \frac{\tanh \frac{\pi(q-\mu)}{a}}{q-\mu}+\int_{0}^{\mu} d q q^{2} \frac{\tanh \frac{\pi(\mu-q)}{a}}{\mu-q}\right],
$$

where the upper limit in the integral was replaced by the cutoff $\Lambda$ for the physical regularization. If the correspondence $\frac{\pi}{a}=\frac{1}{2 T}$ between the acceleration $a$ and temperature $T$ is taken into consideration, this result exactly corresponds to the well known formula for the critical curve in the usual CSC theory at finite temperature [11, 17]. Let us again give a rough estimate of the order of the critical acceleration using the numerical results of [17]. By taking their value of the critical temperature on the transition curve for color superconductivity $T_{c}=40 \mathrm{MeV}$, and the chemical potential $\mu=0.4 \mathrm{GeV}$, we find for the critical acceleration the following estimate $a_{c}=2 \pi T_{c}=2 \pi \times 0.04 \mathrm{GeV}=7.5 \times 10^{34}$ $\mathrm{cm} / \mathrm{s}^{2}$, which differs from the critical acceleration for restoration of chiral symmetry by a factor 4 .

\section{SUMMARY AND CONCLUSIONS}

We have investigated the role of the thermalization effect by the acceleration of an observer for the restoration of chiral and color symmetries in quark matter at finite density in the framework of the NJL model. For this aim, the effective potential and the gap equations both for the chiral and diquark condensates have been analytically derived, and on this basis the values of the critical acceleration, where the chiral or color symmetry is restored, have been determined. Obviously, the acceleration plays here the role of the temperature, as if the system is placed into a thermostat. In particular, we have demonstrated that the results obtained with finite acceleration at the critical points are quite similar to those with the ordinary thermal situation, describing phase transitions with restoration of chiral or color symmetry at finite temperature and chemical potential.

The dependence of chiral and color properties of the quark matter on the acceleration of the observer may be useful in the physics of black holes, where the Rindler metric can be considered as an approximation for the description of the surface gravitational fields[34]. Moreover, the investigation of the influence of strong gravitational fields, such as in compact stars, on the diquark condensation and thus on the possible existence of color superconductivity in the core of the compact stars, is also of great importance. Further investigations in this direction are under way, and their results will appear in subsequent publications.

\section{Acknowledgments}

One of the authors (V.Ch.Zh.) gratefully acknowledges the hospitality of Prof. M. Mueller-Preussker and his colleagues at the particle theory group of the Humboldt University extended to him during his stay there. This work 
was supported by DAAD.

[1] S. W. Hawking, Commun. Math. Phys. 43, 199 (1975); S. A. Fulling, Phys. Rev. D7, 2850 (1973); P. C. W. Davies, J. Phys. A8, 609 (1975); W. G. Unruh, Phys. Rev. D14, 870 (1976); S. Takagi, Prog. Theor. Phys. Suppl. 88, 1 (1986).

[2] W.G. Unruh and N. Weiss, Phys. Rev. D29, 1656 (1984).

[3] Y.K. Lau, Phys. Rev. D53, 3178 (1996).

[4] N.B. Narozhny, V.D. Mur, and A.M. Fedotov, Phys. Lett. A 315, 169 (2003).

[5] D. Kharzeev and K. Tuchin, Nucl. Phys. A753, 316 (2005); hep-ph/0501234.

[6] B.C. Barrois, Nucl. Phys. B 129, 390 (1977).

[7] S.C. Frautschi, "Asymptotic freedom and color superconductivity in dense quark matter", in Proceedings of the Workshop on Hadronic Matter at Extreme Energy Density, Ed., N. Cabibbo, Erice, Italy (1978).

[8] D. Bailin and A. Love, Phys. Rept. 107, 325 (1984).

[9] D.T. Son, Phys. Rev. D 59, 094019 (1999); D.K. Hong, Nucl. Phys. B 582, 451 (2000); S.D.H. Hsu and M. Schwetz, Nucl. Phys. B 572, 211 (2000); R.D. Pisarsky and D.H. Rischke, Phys. Rev. D 61, 074017 (2000); I.A. Shovkovy and L.C.R. Wijewardhana, Phys. Lett. B 470, 189 (1999).

[10] K. Rajagopal and E. Shuster, Phys. Rev. D 62, 085007 (2000).

[11] R. Rapp, T. Schäfer, E.A. Shuryak and M. Velkovsky, Phys. Rev. Lett. 81, 53 (1998); Ann. Phys. 280, 35 (2000); M. Alford, K. Rajagopal and F. Wilczek, Phys. Lett. B 422, 247 (1998).

[12] Y. Nambu and G. Jona-Lasinio, Phys. Rev. 122, 345 (1961); ibid. 124, 246 (1961); V. G. Vaks and A. I. Larkin, ZhETF 40, 282 (1961).

[13] D. Ebert and M.K. Volkov, Yad. Fiz. 36 (1982) 1265 ; Z. Phys. C 16, 205 (1983); D. Ebert and H. Reinhardt, Nucl. Phys. B 271, 188 (1986); D. Ebert, H. Reinhardt and M.K. Volkov, Progr. Part. Nucl. Phys. 33, 1 (1994).

[14] T. Hatsuda and T. Kunihiro, Phys. Rep. 247, 221 (1994).

[15] M. Buballa, Phys. Rep. 407, 205 (2005); G. Nardulli, Riv. Nuovo Cim. 25N3, 1 (2002).

[16] T. Inagaki, T. Muta and S.D. Odintsov, Progr. Theor. Phys. Suppl. 127, 93 (1997).

[17] T.M. Schwarz, S.P. Klevansky and G. Papp, Phys. Rev. C 60, 055205 (1999).

[18] K.G. Klimenko and A.S. Vshivtsev, JETP Lett. 64, 338 (1996); A.S. Vshivtsev, V.Ch. Zhukovsky and K.G. Klimenko, JETP 84, 1047 (1997); A.S. Vshivtsev, M.A. Vdovichenko and K.G. Klimenko, JETP 87, 229 (1998).

[19] S.P. Klevansky and R.H. Lemmer, Phys. Rev. D 39, 3478 (1989); H. Suganuma and T. Tatsumi, Ann.of Phys.208, 470 (1991); T. Inagaki, S.D. Odintsov and Yu.I. Shil'nov, Int. J. Mod. Phys. A 14, 481 (1999); E. Gorbar, Phys. Lett. B 491, 305 (2000).

[20] M.A. Vdovichenko, A.S. Vshivtsev and K.G. Klimenko, Phys. Atom. Nucl. 63, 470 (2000); D. Ebert, K.G. Klimenko, M.A. Vdovichenko and A.S. Vshivtsev, Phys. Rev. D 61, 025005 (2000); D. Ebert and K. G. Klimenko, Nucl. Phys. A 728, 203 (2003).

[21] D. Ebert, V.V. Khudyakov, V.Ch. Zhukovsky, and K.G. Klimenko, Phys. Rev. D 65, 054024 (2002); D. Ebert, K.G. Klimenko, H. Toki, and V.Ch. Zhukovsky, Prog. Theor. Phys. 106, 835 (2001).

[22] M. Alford, K. Rajagopal and F. Wilczek, Nucl. Phys. B 537, 443 (1999); K. Langfeld and M. Rho, Nucl. Phys. A 660, 475 (1999).

[23] J. Berges and K. Rajagopal. Nucl. Phys. B 538, 215 (1999).

[24] M. Alford, hep-ph/0102047; B.O. Kerbikov, hep-ph/0110197.

[25] T. Ohsaku, Phys. Lett. B599, 102 (2004).

[26] X. Huang, X. Hao, and P. Zhuang, hep-ph/0602186.

[27] D. R. Brill and J. A. Wheeler, Rev. Mod. Phys. 29, 465 (1957); L. Parker and D. J. Toms, Phys. Rev. D29, 1584 (1984).

[28] P. Candelas and D. Deutsch, Proc. R. Soc. London, Ser. A 362, 251 (1978); H. Terashima, Phys. Rev. D60, 084001 (1999).

[29] D. Ebert, L. Kaschluhn, and G. Kastelewicz, Phys. Lett. B 264, 420 (1991).

[30] T.D. Lee, Nucl. Phys. B264, 437 (1986); R. Friedberg, T.D. Lee, and Y. Pang, ibid. B276, 549 (1986).

[31] A. A. Abrikosov, L. P. Gor'kov, and I. E. Dzyaloshinskii, Methods of Quantum Field Theory in Statistical Physics (Dover, New York, 1963); J. I. Kapusta, Finite-temperature Field Theory (Cambridge University Press, Cambridge, 1989).

[32] The most general four-fermion interaction would include additional vector and axial-vector $(\bar{q} q)$ as well as pseudo-scalar, vector and axial-vector-like $(q q)$-interactions. For our goal of studying the effect of acceleration on the competition of quark and diquark condensates, the interaction structure of (8) is, however, sufficiently general.

[33] If $\langle\vec{\pi}\rangle \neq 0$ then one would have spontaneous breaking of parity. For strong interactions parity is, however, a conserved quantum number, justifying the assumption $\langle\vec{\pi}\rangle=0$.

[34] Of course, massive black holes with masses $M \gtrsim M_{\odot}$ have a Hawking temperature $T_{H} \lesssim 10^{-6} \mathrm{~K}$, which is much smaller than the temperature of restoration of symmetry, $T_{c} \sim 10^{12} \mathrm{~K}$. On the other hand, primordial black holes with masses $M \sim 10^{12} \mathrm{~kg}$ would lead to a temperature $T_{H} \sim 10^{12} \mathrm{~K}$ of comparable order of magnitude. 